Thus, with respect to the coordinate system $w(\zeta)$, the product function of $G$ is of class $C^{2}$ and hence $G$ is a local Lie group.

Proof of The Theorem. When $G$ has the discrete center, Lemma 5 shows that $G$ is a local Lie group.

When $G$ has the non-discrete center $N$, by Lemma $4, N$ is an abelian Lie group, and by Lemma 5 again, $G / N$ is a local Lie group. Then we can introduce a canonical coordinate of the second kind by $x_{1}^{* \lambda_{1}}, \ldots, x_{m}^{* \lambda_{m}}$, where $x_{i}^{* \lambda_{i}}(i=1,2, \cdots, m)$ are one-parameter subgroups of $G / N$. Take $x_{i}$ of $G$ from the coset $x_{i}^{*}$ for each $i$, we can easily show that the set $M=\left\{y ; y=x_{1}^{\lambda_{1}} \cdots x_{m}^{\lambda_{m}},\left|\lambda_{i}\right| \leqq 1\right\}$ satisfies the condition (3) of Lemma 6 . Consequently, by Lemma $6, G$ is a local Lie group. This completes our proof.

\title{
REFERENCES
}

1. Garrett Birkhoff, Analytical groups, Trans. Amer. Math. Soc. vol. 43 (1934) pp. 61-101.

2. P. A. Smith, Foundation of Lie groups, Ann. of Math. vol. 48 (1947).

3. K. Iwasawa, On some types of topological groups, Ann. of Math. vol. 50 (1949).

4. L. Pontrjagin, Topological groups, Princeton, 1939.

TOKYO INSTITUTE OF TECHNOLOGY

\section{NOTE ON A THEOREM OF KOKSMA}

\section{WM. J. LEVEQUE ${ }^{1}$}

In 1935 Koksma [2] $]^{2}$ showed, among other things, that the sequence $x, x^{2}, x^{3}, \cdots$ is uniformly distributed $(\bmod 1)$ for almost all $x>1$; that is, that if $N(n, \alpha, \beta, x)$ denotes the number of elements $x^{j}$ of the sequence $x, x^{2}, \cdots, x^{n}$ for which

$$
0 \leqq \alpha \leqq x^{j}-\left[x^{j}\right]<\beta \leqq 1,
$$

then

$$
\lim _{n \rightarrow \infty} \frac{N(n, \alpha, \beta, x)}{n}=\beta-\alpha
$$

Presented to the Society, September 10,1948, under the title $A$ metric theorem on uniform distribution (mod 1); received by the editors January 24, 1949 and, in revised form, February 10, 1949.

1 The author is indebted to Professor Mark Kac for his help in connection with this paper.

2 Numbers in brackets refer to the bibliography at the end of the paper. 
for almost all $x>1$. The purpose of this note is to provide another proof of this theorem, based on a lemma used in a recent paper [1] of $\mathrm{Kac}$, Salem, and Zygmund on quasi-orthogonal functions. The assertion is contained in the more general theorem:

TheOREM 1. Let $g(x, n)$ be any function of the real variable $x$ and the positive integral variable $n$ with the following properties in the interval $a<x<b$ :

(i) $d g / d x, d^{2} g / d x^{2}$ exist,

(ii) $g^{\prime}(x, j)-g^{\prime}(x, k)$ is monotonic, and is different from zero for $j \neq k$,

(iii) for $x=a$ and $x=b$, the inequality

$$
\left|g^{\prime}(x, j)-g^{\prime}(x, k)\right| \geqq C|j-k| \cdot
$$

is fulfilled for some $C>0,0<\epsilon \leqq 1$.

Then the sequence $g(x, 1), g(x, 2), \cdots$ is uniformly distributed $(\bmod 1)$ for almost all $x \in(a, b)$.

This theorem is weaker than Theorem 3 of Koksma's paper, but it is easily verified that the conditions of the theorem are satisfied for

$$
\begin{aligned}
& g(x, n)=x^{n}, \quad 1 \leqq a<b, \\
& g(x, n)=n^{t} x, \quad \text { every } a, b \quad(t \text { a positive integer), } \\
& g(x, n)=n^{x}, \quad 1 \leqq a<b, \\
& g(x, n)=x^{M(n)}, \quad 1 \leqq a<b,
\end{aligned}
$$

where $M(n)$ is positive and such that $|M(j)-M(k)| \geqq N$ for some $N>0$ and all $j \neq k$. This last case is Theorem 2 of Koksma's paper.

We use the following specialization of Lemma 1 of [1]: Let $g(x, n)(n=1,2, \cdots)$ be any sequence of real continuous functions in $(a, b)$, and let $m$ be an integer different from zero. Suppose that for all positive integers $j, k$ with $j \neq k$, we have

$$
\left|\int_{a}^{b} e^{2 \pi i m(\theta(x, j)-\theta(x, k))} d x\right| \leqq \frac{C_{1}}{|j-k| \cdot}
$$

for some $\epsilon$ with $0<\epsilon \leqq 1$. Then the series

$$
\sum_{n=1}^{\infty} \frac{e^{2 \pi i m g(x, n)}}{n^{1-\delta}}
$$

converges almost everywhere in $(a, b)$ for every $\delta<\epsilon / 2$.

(Lemma 1 of [1] is stated for real-valued functions; in the case of complex-valued functions the integrand should be replaced by $f_{j}(x) \bar{f}_{k}(x)$, where $\bar{f}$ is the complex conjugate of $f$.) 
We shall show that for a function $g$ satisfying (i), (ii), and (iii) of the theorem, the conditions of the lemma are also satisfied for each $m$. The validity of the theorem then follows from Weyl's criterion $[3$, p. 91] for uniform distribution $(\bmod 1)$ upon noting that the convergence of $\sum a_{n} / n$ implies that

$$
\lim _{N \rightarrow \infty} \frac{1}{N} \sum_{n=1}^{N} a_{n}=0 .
$$

Assume (i), (ii), and (iii). Then

$$
\begin{aligned}
& \left|\int_{a}^{b} \exp \{2 \pi i m(g(x, j)-g(x, k))\} d x\right| \\
& =\left|\int_{a}^{b} \exp \{2 \pi i m(g(x, j)-g(x, k))\} \cdot \frac{g^{\prime}(x, j)-g^{\prime}(x, k)}{g^{\prime}(x, j)-g^{\prime}(x, k)} d x\right| \\
& \leqq \frac{1}{2 \pi|m|}\left(\left|\left[\frac{\exp \{2 \pi i m(g(x, j)-g(x, k))\}}{g^{\prime}(x, j)-g^{\prime}(x, k)}\right]_{a}^{b}\right|\right. \\
& +\mid \int_{a}^{b} \exp \{2 \pi i m(g(x, j) \\
& \left.-g(x, k))\} \frac{d}{d x}\left(g^{\prime}(x, j)-g^{\prime}(x, k)\right)^{-1} d x \mid\right) \\
& \leqq \frac{1}{2 \pi|m|}\left(\frac{1}{\left|g^{\prime}(a, j)-\left(g^{\prime} a, k\right)\right|}+\frac{1}{\left|g^{\prime}(b, j)-g^{\prime}(b, k)\right|}\right. \\
& \left.+\int_{a}^{b}\left|\frac{d}{d x}\left(g^{\prime}(x, j)-g^{\prime}(x, k)\right)^{-1}\right| d x\right) \text {. }
\end{aligned}
$$

By (ii), this is

$$
\begin{aligned}
& \leqq \frac{1}{2 \pi|m|}\left(\frac{1}{\mid g^{\prime}(a, j)-\left(g^{\prime}(a, k) \mid\right.}+\frac{1}{\left|g^{\prime}(b, j)-g^{\prime}(b, k)\right|}\right. \\
& \left.+\left|\int_{a}^{b} \frac{d}{d x}\left(g^{\prime}(x, j)-g^{\prime}(x, k)\right)^{-1} d x\right|\right) \\
& \leqq \frac{1}{\pi|m|}\left(\frac{1}{\left|g^{\prime}(a, j)-g^{\prime}(a, k)\right|}+\frac{1}{\left|g^{\prime}(b, j)-g^{\prime}(b, k)\right|}\right),
\end{aligned}
$$

and this, by (iii), is

$$
\leqq \frac{2}{C \pi|m| \cdot|j-k| \epsilon}
$$


so that the assumptions of the lemma hold with $C_{1}=2 / C \pi|m|$.

Actually, we have proved considerably more than is stated in Theorem 1, at least for the special functions $g(x, n)$ cited above. In each of these cases, the hypothesis of Theorem 1 holds with $\epsilon=1$. It follows that for every integer $m \neq 0$, the series

$$
\sum_{n=1}^{\infty} \frac{e^{2 \pi i m_{\theta}(x, n)}}{n^{1 / 2+\theta}}
$$

converges for almost all $x \in(a, b)$. Using Abel's partial summation formula we deduce the following theorem.

THEOREM 2. Under the assumptions of Theorem 1,

$$
\sum_{n=1}^{N} e^{2 \pi i m o(x, n)}=o\left(N^{1 / 2+\theta}\right),
$$

for every integer $m \neq 0$ and for almost all $x \in(a, b)$.

This is a much stronger statement than an assertion about uniform distribution. In view of its generality it is remarkably close to best possible, since it is known [4] that for $g(x, n)=x n^{2}$, this sum is not $o\left(N^{1 / 2}\right)$ for any irrational $x$.

\section{BIBLIOGRAPHY}

1. M. Kac, R. Salem, and A. Zygmund, $A$ gap theorem, Trans. Amer. Math. Soc. vol. 63 (1948) pp. 235-243.

2. J. F. Koksma, Ein mengentheoretischer Satz über die Gleichverteilung modulo Eins, Compositio Mathematica vol. 2 (1935) pp. 250-258.

3. - Diophantische Approximationen, Ergebnisse der Mathematik vol.4, no. 4, 1936.

4. G. H. Hardy and J. E. Littlewood, The trigonometrical series associated with the elliptic $\theta$-functions, Acta Math. vol. 37 (1914) pp. 193-239.

HARVARD UNIVERSITY 